\title{
Excitation and recombination dynamics of vacancy-related spin centers in silicon carbide
}

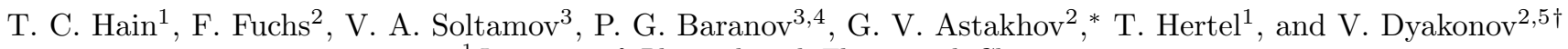 \\ ${ }^{1}$ Institute of Physical and Theoretical Chemistry, \\ Julius-Maximilian University of Würzburg, 97074 Würzburg, Germany \\ ${ }^{2}$ Experimental Physics VI, Julius-Maximilian University of Würzburg, 97074 Würzburg, Germany \\ ${ }^{3}$ Ioffe Physical-Technical Institute, 194021 St. Petersburg, Russia \\ ${ }^{4}$ St. Petersburg State Polytechnical University, 195251 St. Petersburg, Russia \\ ${ }^{5}$ Bavarian Center for Applied Energy Research (ZAE Bayern), 97074 Würzburg, Germany
}

(Dated: October 4, 2018)

\begin{abstract}
We generate silicon vacancy related defects in high-quality epitaxial silicon carbide layers by means of electron irradiation. By controlling the irradiation fluence, the defect concentration is varied over several orders of magnitude. We establish the excitation profile for optical pumping of these defects and evaluate the optimum excitation wavelength of $770 \mathrm{~nm}$. We also measure the photoluminescence dynamics at room temperature and find a monoexponential decay with a characteristic lifetime of $6.1 \mathrm{~ns}$. The integrated photoluminescence intensity depends linear on the excitation power density up to $20 \mathrm{~kW} / \mathrm{cm}^{2}$, indicating a relatively small absorption cross section of these defects.
\end{abstract}

PACS numbers: 78.55.-m, 78.47.jd, 61.72.jd

Silicon carbide $(\mathrm{SiC})$ has recently been suggested for deployment in various quantum technologies [1] 8 . In particular, silicon vacancy $\left(\mathrm{V}_{\mathrm{Si}}\right)$ related defects can be used as coherently controllable [4, 9] and selectively addressable 6, 10, qubits, characterized by long spin coherence and spin-lattice relaxation times even at room temperature. These atomic-scale qubits are also attractive for local sensing of magnetic [11] and electric [12] fields as well as temperature [11. Another potential use is as a low-noise quantum microwave amplifier based on the maser effect 13. Furthermore, $\mathrm{SiC}$ is a technologically friendly material, used in various devices (LED, MOSFETS, MEMS, sensors). This facilitates attractive opportunities for electrical control of these vacancy-related spin centers [14, 15].

In all these appealing applications, an optical pumping scheme of the high-spin ground state of $\mathrm{V}_{\mathrm{Si}}$-related defects $(S=1$ or $S=3 / 2)$ [13, 16 22] is exploited. This pumping scheme is assumed to be similar to that of the nitrogen-vacancy (NV) defect in diamond [23. In spite of this similarity, the optical properties of the $\mathrm{V}_{\mathrm{Si}}$-related defects in $\mathrm{SiC}$ have not been measured in detail. It is worth noting that another vacancy centers in $\mathrm{SiC}$, associated with the so-called $\mathrm{AB}$ lines, have been demonstrated to be very efficient single photon sources 24. In these single-defect experiments, the positively charged configuration of these centers has been investigated, which has low-spin ground state $(S=1 / 2)$. Therefore, it is unclear whether their quantum control is possible in the same manner as for the $\mathrm{V}_{\mathrm{Si}}$-related defects in $\mathrm{SiC}$ or the $\mathrm{NV}$ defect in diamond.

In this Letter, we measure excitation spectrum and recombination dynamics of $\mathrm{V}_{\mathrm{Si}}$ in $4 \mathrm{H}-\mathrm{SiC}$ at room temperature. The crystal structure of polytype $4 \mathrm{H}$ is shown in Fig. 1(a). There are four $\mathrm{Si}$ and four $\mathrm{C}$ atoms per unit cell with stacking sequence $\mathrm{ABCB}$ (where $\mathrm{A}, \mathrm{B}$ and $\mathrm{C}$ denote close-packed atomic planes). The broken bonds with missing Si atom result in the appearance of additional energy states and the electron density is mostly localized at the first- $(\mathrm{C})$ and second-neighbor $(\mathrm{Si})$ atoms to this $\mathrm{V}_{\mathrm{Si}}$ vacancy. According to the density functional theory (DFT) calculations 25, the highest occupied state and the lowest (partially) unoccupied states lie deep in the forbidden gap of $\mathrm{SiC}$, which is $E_{g}=3.23 \mathrm{eV}$ at room temperature. For this reason, these defects can be detected by their characteristic photoluminescence (PL) in the spectral range shifted towards longer wavelengths with respect to the fundamental emission/absorption edge (ca. $400 \mathrm{~nm}$ in $4 \mathrm{H}-\mathrm{SiC}$ ). At cryogenic temperatures, the socalled zero-phonon lines (ZPLs) are observed, distinct in their optical transition energies. In highly homogeneous samples, these ZPLs are very sharp with full width at half maximum (FWHM) of a few $\mu \mathrm{eV}$ (ref. 6). With rising temperature, the ZPLs are significantly broadened due to interaction with thermally excited phonons and their FWHM is approximately $100 \mathrm{meV}$ at $T=300 \mathrm{~K}$.

In $4 \mathrm{H}-\mathrm{SiC}$ there are two non-equivalent lattice sites and, therefore, there are two different $\mathrm{V}_{\mathrm{Si}}$ defects with corresponding ZPLs labeled as V1 and V2 (ref. 26). These defects are probably perturbed by carbon vacancies [7, which are not shown in Fig. 1(a) for simplicity. When a silicon and carbon vacancies are situated along a chemical bond, a divacancy $\left(\mathrm{V}_{\mathrm{Si}^{-}}-\mathrm{V}_{\mathrm{C}}\right)$ is formed [1, 22, having its own ZPL.

The 4H-SiC samples investigated in this Letter have been purchased from CREE. A nominally undoped (residual n-type doping $5.0 \times 10^{14} \mathrm{~cm}^{-3}$ ) layer of $110 \mu \mathrm{m}$ thickness was epitaxially grown on a 2 -inch n-type $4 \mathrm{H}$ $\mathrm{SiC}$ wafer $\left(396 \mu \mathrm{m}\right.$ thickness, $7.84^{\circ}$ off-axis orientation). The layer is conserved by a 5 - $\mu$ m-thick n-type $4 \mathrm{H}-\mathrm{SiC}$ 


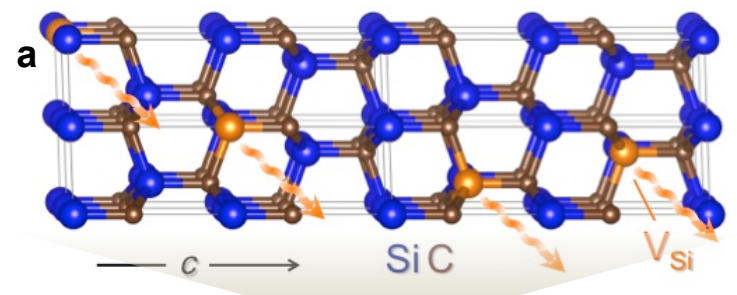

b

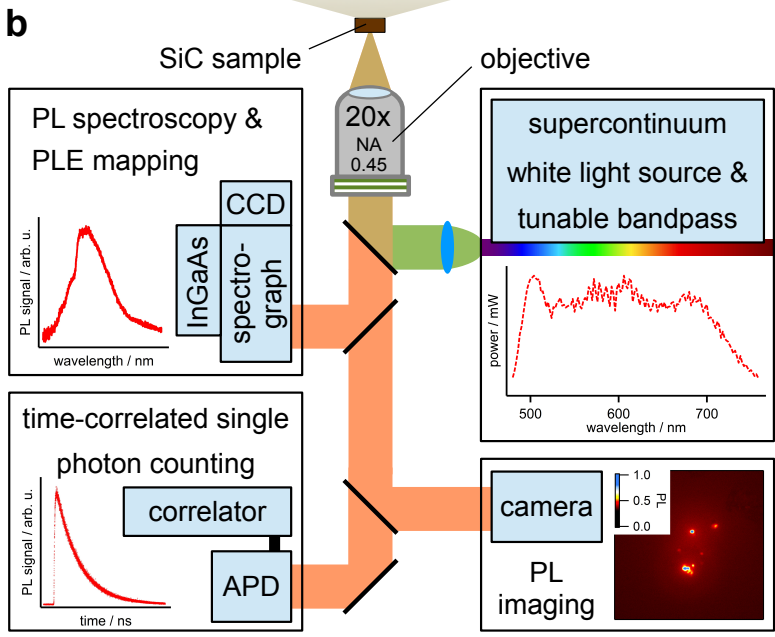

FIG. 1: (a) Crystal structure of $4 \mathrm{H}-\mathrm{SiC}$ and silicon vacancy defects $\left(\mathrm{V}_{\mathrm{Si}}\right)$. The wavy arrows indicate the near infrared emission of $\mathrm{V}_{\mathrm{Si}}$. (b) A scheme of the experimental setup for spatial imaging, spectroscopical and time-correlated measurements.

layer $\left(1.2 \times 10^{16} \mathrm{~cm}^{-3}\right)$ and a 2 - $\mu$ m-thick p-type $4 \mathrm{H}$ $\mathrm{SiC}$ layer $\left(6.0 \times 10^{16} \mathrm{~cm}^{-3}\right)$. The wafer was diced in $4 \mathrm{~mm} \times 2 \mathrm{~mm}$ pieces. In order to create $\mathrm{V}_{\mathrm{Si}}$-related defects the samples were irradiated with $0.9 \mathrm{MeV}$ electrons to different fluences.

First, we present spatial imaging of defects in our $\mathrm{SiC}$ samples [our microscopy setup is schematically sketched in Fig. 1 (b)]. The sample is placed in an inverted Ti-U microscope and the PL is excited and collected through a 20x CFI Super Plan Fluor ELWD objective with N.A. $=0.45$ (Nikon). The excitation light from a SuperK EXTREME (EXR-15) supercontinuum laser (NKT Photonics) was defocused onto a spot of approximately $50 \mu \mathrm{m}$ in diameter. The PL image in the confocal plane of the objective is recorded by a Clara interline CCD camera (Andor), with one CCD pixel $(6.45 \mu \mathrm{m})$ corresponding to $323 \mathrm{~nm}$ at the sample position. The difraction-limited spatial resolution of the setup is about $1.3 \mu \mathrm{m}$. We use a wide-band excitation $(480-740 \mathrm{~nm})$ for imaging, and the emission is detected for longer wavelengths $(\lambda>760 \mathrm{~nm})$.

To measure PL spectra we use a Shamrock SR-303i spectrograph (Andor) together with either a Newton CCD camera (DU920P-OE) for detection from 760 to $1000 \mathrm{~nm}$ or a iDus InGaAs array detector (DU491A-1.7) for detection from 1000 to $1500 \mathrm{~nm}$ [Fig. 1(b)]. This con-
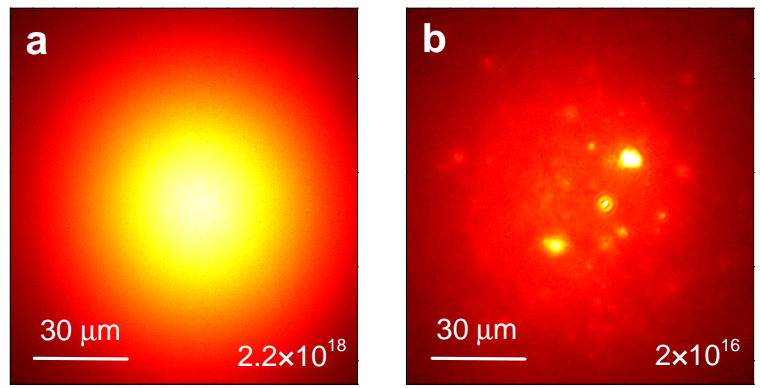

FIG. 2: (a) Spatial imaging of the $\mathrm{V}_{\mathrm{Si}}$-related PL in a strongly irradiated sample (irradiation fluence $2.2 \times 10^{18} \mathrm{e} / \mathrm{cm}^{2}$ ). (b) The same as (a) but in a weakly irradiated sample (irradiation fluence $2 \times 10^{16} \mathrm{e} / \mathrm{cm}^{2}$ ). Note, the intensity scales in (a) and (b) are different.

figuration provides the spectral resolution of ca. $0.6 \mathrm{~nm}$. For photoluminescence excitation (PLE) measurements, the laser wavelength is selected from the supercontinuum spectrum with a step of $2 \mathrm{~nm}$ using a SuperK VARIA bandpass filter (NKT Photonics). In low-temperature experiments $(T=10 \mathrm{~K})$, the sample is mounted in a coldfinger MicrostatHe (Oxford Instruments) and placed in a LabRAM system for spectroscopy (Horiba Scientific). In this case we use a He-Ne laser $(633 \mathrm{~nm})$ for excitation.

Figure 2 shows PL images of two samples irradiated with electrons to different fluences. The wavelength range detected by the PL microscope setup is associated with emission from color centers (below we demonstrate that these colour centres are the $\mathrm{V}_{\mathrm{Si}}$ defects). At a high irradiation fluence $\left(2.2 \times 10^{18} \mathrm{e} / \mathrm{cm}^{2}\right)$, the PL intensity appears to closely follow the excitation profile which suggests that emission is from a homogeneous distribution of color centers [Fig. 2[a)]. At a much lower irradiation fluence $\left(2 \times 10^{16} \mathrm{e} / \mathrm{cm}^{2}\right)$ this profile splits up into several more strongly emissive fragments [Fig. 2(b)]. Some of these fragments have sub- $\mu \mathrm{m}$ size and reveal diffraction rings. Neither of these is observed in as-grown samples suggesting that these color centers were produced by electron irradiation.

The room-temperature PL spectra are presented in Fig. 3(a). With increasing electron irradiation from $2.2 \times 10^{18} \mathrm{e} / \mathrm{cm}^{2}$ to $7.5 \times 10^{18} \mathrm{e} / \mathrm{cm}^{2}$ the integrated PL intensity increases ca. by a factor of three, suggesting that the defect concentration is proportional to the irradiation fluence. We observe PL in the spectral range from 850 to $1050 \mathrm{~nm}$, which is characteristic for $\mathrm{V}_{\mathrm{Si}}$ in $\mathrm{SiC}$ at room temperature [14. On the other hand, we do not observe PL in the spectral range from 1050 to $1350 \mathrm{~nm}$, where $\mathrm{PL}$ from $\mathrm{V}_{\mathrm{Si}^{-}}-\mathrm{V}_{\mathrm{C}}$ is expected [4]. By cooling down to $T=10 \mathrm{~K}$ we resolve four ZPLs in the PL spectrum [Fig. 3(b)]. The V1 ZPL at $\lambda=861.4 \mathrm{~nm}$ dominates in the spectrum, unambiguously proving that we generate and probe the $\mathrm{V}_{\mathrm{Si}}$ defects.

In oder to find the optimal excitation conditions we 

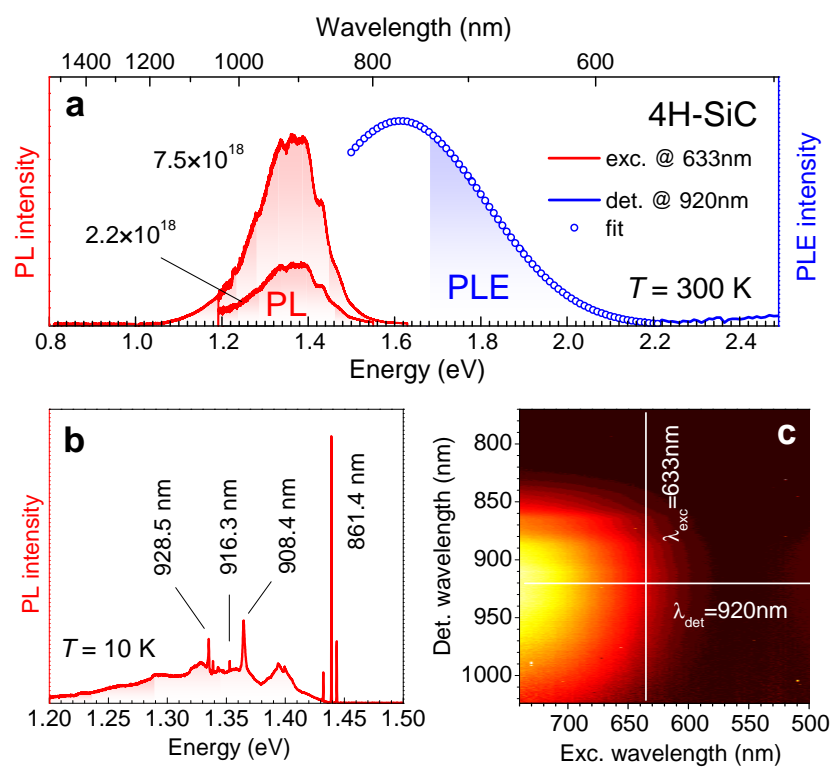

FIG. 3: (a) Room-temperature PL and PLE spectra of $\mathrm{V}_{\mathrm{Si}}$ in $4 \mathrm{H}-\mathrm{SiC}$ for different irradiation fluences. The Gaussian fit of the PLE spectrum is shown by open circles. (b) A low-temperature PL spectrum (irradiation fluence $7.5 \times$ $\left.10^{18} \mathrm{e} / \mathrm{cm}^{2}\right)$. The $\mathrm{V} 1$ and $\mathrm{V} 2 \mathrm{ZPLs}$ of $\mathrm{V}_{\mathrm{Si}}$ are observed at $861.4 \mathrm{~nm}$ and $916.3 \mathrm{~nm}$, respectively. (c) A $2 \mathrm{D}$ scan of the excitation and detection wavelengths at room temperature.

perform PLE mapping experiments. First, we ascertain that the PL spectrum is independent of the excitation wavelength [Fig. 3(c)]. A PLE spectrum, detected at the PL maximum $(\lambda=920 \mathrm{~nm})$ and corrected for the laser supercontinuum spectrum, is presented in Fig. 3(a). With decreasing wavelength $(\lambda<760 \mathrm{~nm})$ the excitation becomes less efficient and for $\lambda<590 \mathrm{~nm}$ the $\mathrm{V}_{\mathrm{Si}} \mathrm{PL}$ is not excited. The corresponding energy $2.1 \mathrm{eV}$ is still significantly smaller than the bandgap in $4 \mathrm{H}-\mathrm{SiC}(3.23 \mathrm{eV})$. According to the Franck-Condon principle, the PLE maximum is shifted towards higher energy with respect to the ZPL. In oder to estimate this maximum, we fit the PLE spectrum in Fig. 3(a). In fact, the PLE spectrum associated with vibronic bands in the crystal has a complex form and is usually asymmetric with respect to the maximum. We find that the highenergy tail of the experimentally obtained PLE spectrum $(E>1.68 \mathrm{eV})$ is perfectly described by the Gaussian in the form $\exp \left(-2\left(\frac{E-E_{\max }}{\Delta}\right)^{2}\right)$ with $E_{\max }=1.61 \mathrm{eV}$ and $\Delta=0.40 \mathrm{eV}$ [open circles in Fig. 3(a)], indicating that inhomogeneous broadening dominates the excitation spectrum. Following this fit, the optimal excitation wavelength is around $770 \mathrm{~nm}$. Interestingly, we observe non-equivalent linewidth of the PL and PLE spectra, expected to be similar from a configuration coordinate diagram approach. A possible explanation could be due to the different interaction with phonons in the ground and excited states. Further understanding requires detailed
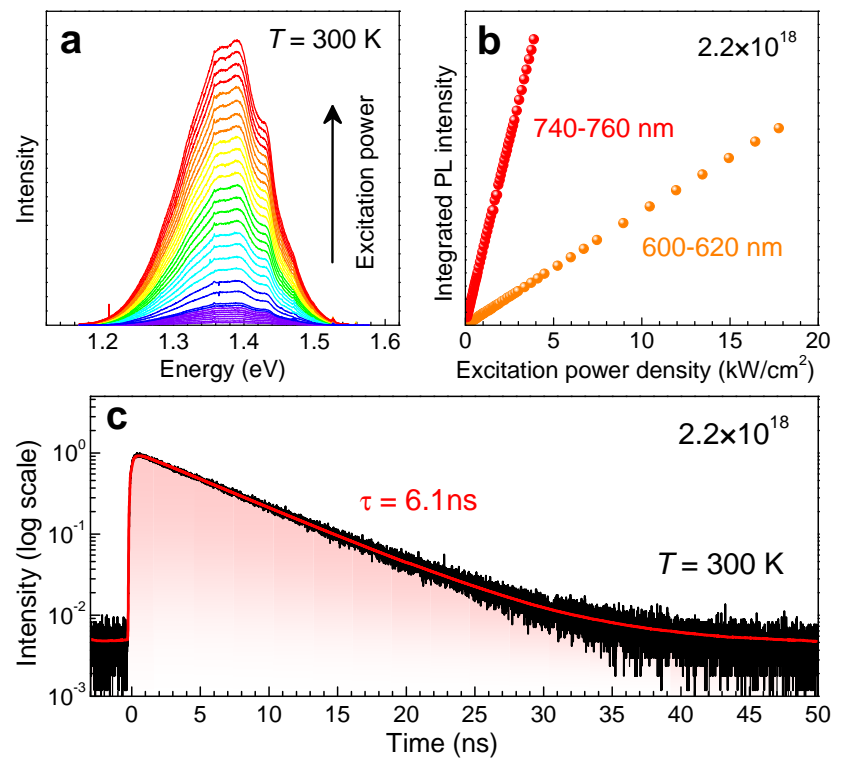

FIG. 4: (a) PL spectra with rising excitation power. (b) Excitation power density dependence of the integrated PL intensity for two different excitation spectral ranges. (c) Monoexponential PL decay of $\mathrm{V}_{\mathrm{Si}}$ with a characteristic lifetime $\tau=6.1 \mathrm{~ns}$.

theoretical consideration, which is beyond the scope of this work.

To estimate the excitation efficiency we measure the integrated PL intensity as a function of excitation power [Fig. 4(a)]. It is presented in Fig. 4(b) for two spectrally different excitations. The excitation in the spectral range $740-760 \mathrm{~nm}$ is more efficient than in $600-620 \mathrm{~nm}$, as expected from the PLE spectrum of Fig. 3(a). We observe perfectly linear dependences on the excitation power density up to $20 \mathrm{~kW} / \mathrm{cm}^{2}(600-620 \mathrm{~nm})$ and $3 \mathrm{~kW} / \mathrm{cm}^{2}$ $(740-760 \mathrm{~nm})$, indicating that all the results reported here are obtained far below the saturation. Remarkably, the saturation power density for the NV defect is about $100 \mathrm{~kW} / \mathrm{cm}^{2}$ (refs. 23, 27). As we do not observe deviation from the linear dependence, this suggests that the absorption cross section of the $\mathrm{V}_{\mathrm{Si}}$ defects is comparable or smaller than that of the NV defect.

Finally, we analyze PL dynamics of $\mathrm{V}_{\mathrm{Si}}$, using timecorrelated single photon counting (TCSPC) measurements. The repetition rate of the pulsed ( $5 \mathrm{ps)}$ excitation is $10 \mathrm{MHz}$ and the spectrally integrated PL signal is detected using a Si single-photon avalanche photodiode and a TCSPC HydraHarp module (PicoQuant) [Fig. 1(b)]. To characterize our setup we measure the instrument response function, using scattered light from the laser in the spectral range from 800 to $840 \mathrm{~nm}$, and obtain the overall time resolution of $50 \mathrm{ps}$.

The decay of the $\mathrm{V}_{\mathrm{Si}} \mathrm{PL}$ after pulsed excitation is presented in Fig. 4(c). The experimental data are perfectly fitted with a monoexponential decay of the form 
$a+b \exp (-t / \tau)$ convoluted with the instrument response function. From the fit we find the PL lifetime $\tau=6.1 \mathrm{~ns}$. This is of an order of magnitude comparable to that of another color center in $\mathrm{SiC}(1.2 \mathrm{~ns}) 24$ and of the NV defect in diamond $(11.7 \mathrm{~ns})$ 27]. This similarity is expected because all these atomic-scale defects are isolated from the bulk bands and their optical transitions are determined by the coupling of the electronic and vibronic states. The transition rate in this case, as a rule, is smaller than for direct band-to-band transitions in semiconductor nanostructures, like colloidal quantum dots 28, and quantum wells [29].

Summarizing, we have performed spatial imaging of the $\mathrm{V}_{\mathrm{Si}}$ defects, generated by electron irradiation in epitaxial 4H-SiC layer, and investigated their optical properties. In particular, we have found the optimal excitation conditions to optically pump these defects and we have measured their PL lifetime. This information is important to optimize quantum control of these defects, as required for various appealing applications.

This work has been supported by the Bavarian Ministry of Economic Affairs, Infrastructure, Transport and Technology as well as by the DFG under grant AS310/4.

* E-mail: astakhov@physik.uni-wuerzburg.de

† E-mail: dyakonov@physik.uni-wuerzburg.de

[1] P. G. Baranov, I. V. Il'in, E. N. Mokhov, M. V. Muzafarova, S. B. Orlinskii, and J. Schmidt, Journal of Experimental and Theoretical Physics Letters 82, 441 (2005).

[2] J. R. Weber, W. F. Koehl, J. B. Varley, A. Janotti, B. B. Buckley, C. G. Van de Walle, and D. D. Awschalom, Proceedings of the National Academy of Sciences 107, 8513 (2010).

[3] P. Baranov, A. Bundakova, A. Soltamova, S. Orlinskii, I. Borovykh, R. Zondervan, R. Verberk, and J. Schmidt, Physical Review B 83, 125203 (2011).

[4] W. F. Koehl, B. B. Buckley, F. J. Heremans, G. Calusine, and D. D. Awschalom, Nature 479, 84 (2011).

[5] A. Gali, Materials Science Forum 717-720, 255 (2012).

[6] D. Riedel, F. Fuchs, H. Kraus, S. Väth, A. Sperlich, V. Dyakonov, A. Soltamova, P. Baranov, V. Ilyin, and G. V. Astakhov, Physical Review Letters 109, 226402 (2012).

[7] P. G. Baranov, V. A. Soltamov, A. A. Soltamova, G. V. Astakhov, and V. D. Dyakonov, Materials Science Forum 740-742, 425 (2013).

[8] S. Castelletto, B. C. Johnson, and A. Boretti, Advanced Optical Materials 1, 609 (2013).

[9] V. Soltamov, A. Soltamova, P. Baranov, and I. Proskuryakov, Physical Review Letters 108, 226402
(2012).

[10] A. L. Falk, B. B. Buckley, G. Calusine, W. F. Koehl, V. V. Dobrovitski, A. Politi, C. A. Zorman, P. X. L. Feng, and D. D. Awschalom, Nature Communications 4, 1819 (2013).

[11] H. Kraus, V. A. Soltamov, F. Fuchs, D. Simin, A. Sperlich, P. G. Baranov, G. V. Astakhov, and V. Dyakonov, arXiv: 1403.7741

[12] A. L. Falk, P. V. Klimov, B. B. Buckley, V. Ivády, I. A. Abrikosov, G. Calusine, W. F. Koehl, A. Gali, and D. D. Awschalom, arXiv:1311.6832

[13] H. Kraus, V. A. Soltamov, D. Riedel, S. Väth, F. Fuchs, A. Sperlich, P. G. Baranov, V. Dyakonov, and G. V. Astakhov, Nature Physics 10, 157 (2014).

[14] F. Fuchs, V. A. Soltamov, S. Väth, P. G. Baranov, E. N. Mokhov, G. V. Astakhov, and V. Dyakonov, Scientific Reports 3, 1637 (2013).

[15] P. V. Klimov, A. L. Falk, B. B. Buckley, and D. D. Awschalom, Physical Review Letters 112, 087601 (2014).

[16] V. S. Vainer and V. A. Il'in, Sov. Phys. Solid State 23, 2126 (1981).

[17] T. Wimbauer, B. Meyer, A. Hofstaetter, A. Scharmann, and H. Overhof, Physical Review B 56, 7384 (1997).

[18] E. Sörman, N. Son, W. Chen, O. Kordina, C. Hallin, and E. Janzén, Physical Review B 61, 2613 (2000).

[19] N. Mizuochi, S. Yamasaki, H. Takizawa, N. Morishita, T. Ohshima, H. Itoh, and J. Isoya, Physical Review B 66, 235202 (2002).

[20] S. Orlinski, J. Schmidt, E. Mokhov, and P. Baranov, Physical Review B 67, 125207 (2003).

[21] I. V. Ilyin, M. V. Muzafarova, E. N. Mokhov, V. I. Sankin, P. G. Baranov, S. B. Orlinskii, and J. Schmidt, Materials Science Forum 527-529, 535 (2006).

[22] N. Son, P. Carlsson, J. ul Hassan, E. Janzén, T. Umeda, J. Isoya, A. Gali, M. Bockstedte, N. Morishita, T. Ohshima, et al., Physical Review Letters 96, 055501 (2006).

[23] F. Jelezko and J. Wrachtrup, physica status solidi (a) 203, 3207 (2006).

[24] S. Castelletto, B. C. Johnson, V. Ivády, N. Stavrias, T. Umeda, A. Gali, and T. Ohshima, Nature Materials 13, 151 (2013).

[25] A. Gali, Journal of Materials Research 27, 897 (2012).

[26] M. Wagner, B. Magnusson, W. Chen, E. Janzén, E. Sörman, C. Hallin, and J. Lindström, Physical Review B 62, 16555 (2000).

[27] C. Kurtsiefer, S. Mayer, P. Zarda, and H. Weinfurter, Physical Review Letters 85, 290 (2000).

[28] V. I. Klimov, A. A. Mikhailovsky, D. W. McBranch, C. A. Leatherdale, and M. G. Bawendi, Science 287, 1011 (2000).

[29] D. R. Yakovlev, J. Puls, G. V. Mikhailov, G. V. Astakhov, V. P. Kochereshko, W. Ossau, J. Nürnberger, W. Faschinger, F. Henneberger, and G. Landwehr, physica status solidi (a) 178, 501 (2000). 